\title{
One-step self-assembled nanomicelles for improving the oral bioavailability of nimodipine
}

This article was published in the following Dove Press journal:

International Journal of Nanomedicine

15 March 2016

Number of times this article has been viewed

Jing-Wen Luo

Zhi-Rong Zhang

Tao Gong

Yao Fu

Key Laboratory of Drug Targeting and Drug Delivery Systems, Ministry of Education, West China School of Pharmacy, Sichuan University, Chengdu, Sichuan, People's Republic of China
Correspondence: Tao Gong; Yao Fu Key Laboratory of Drug Targeting and Drug Delivery Systems, Ministry of Education, West China School of Pharmacy, Sichuan University, No 17 Third Section of Renmin South Road, Chengdu, Sichuan 61004I, People's Republic of China Tel/fax+862885501615 Email gongtaoy@126.com; yfu4@scu.edu.cn
Abstract: Our study aimed to develop a self-assembled nanomicelle for oral administration of nimodipine (NIM) with poor water solubility. Using Solutol ${ }^{\circledR}$ HS15, the NIM-loaded selfassembled nanomicelles displayed a near-spherical morphology with a narrow size distribution of $12.57 \pm 0.21 \mathrm{~nm}$ (polydispersity index $=0.071 \pm 0.011$ ). Compared with Nimotop ${ }^{\circledR}$ (NIM tablets), the intestinal absorption of NIM from NIM nanomicelle in rats was improved by 3.13- and 2.25 -fold in duodenum and jejunum at 1 hour after oral administration. The cellular transport of NIM nanomicelle in Caco-2 cell monolayers was significantly enhanced compared to that of Nimotop ${ }^{\circledR}$. Regarding the transport pathways, clathrin, lipid raft/caveolae, and macropinocytosis mediated the cell uptake of NIM nanomicelles, while P-glycoprotein and endoplasmic reticulum/ Golgi complex (ER/Golgi) pathways were involved in exocytosis. Pharmacokinetic studies in our research laboratory have showed that the area under the plasma concentration-time curve $\left(\mathrm{AUC}_{0-\infty}\right)$ of NIM nanomicelles was 3.72-fold that of Nimotop ${ }^{\mathbb{B}}$ via oral administration in rats. Moreover, the NIM concentration in the brain from NIM nanomicelles was dramatically improved. Therefore, Solutol ${ }^{\circledR}$ HS15-based self-assembled nanomicelles represent a promising delivery system to enhance the oral bioavailability of NIM.

Keywords: nanomicelles, stability, nimodipine, oral bioavailability, transport mechanism

\section{Introduction}

Nimodipine (NIM) is a dihydropyridine calcium antagonist, which dilates cerebral arterioles and increases the cerebral blood flow in animals and humans. ${ }^{1}$ NIM shows desirable pharmacological activities in the treatment of various cerebrovascular disorders, including delayed ischemic neurological deficits, cerebral malaria, cerebral vasospasm, acute ischemic stroke, and migraines. ${ }^{2}$ However, several unfavorable properties greatly limit the clinical application of NIM. First, the oral bioavailability of NIM remains low $(\sim 13 \%)$ due to the significant first-pass effect in liver, whereby only a small fraction of the administered dose can escape extensive liver metabolism and be delivered to the brain. ${ }^{3}$ Moreover, NIM is poorly water soluble $(2.29 \mu \mathrm{g} / \mathrm{mL}$ at $37^{\circ} \mathrm{C}$ ). An injection of NIM containing $40 \%$ solvent mixture, ie, $23.7 \%(\mathrm{v} / \mathrm{v})$ ethanol and 17\% (v/v) polyethylene glycol (PEG)-400, was proven to achieve a sufficient NIM concentration after in vivo administration. ${ }^{4}$ NIM ethanol injections were reported to cause pain and inflammation at the injection site. Furthermore, when NIM injection is diluted with injection solutions, crystallization may occur, which is often life threatening to patients. ${ }^{5}$ Nimotop $^{\circledR}$ (NIM tablets) has been marketed by Bayer AG, Leverkusen, Germany as an oral dosage form of NIM; however, the recommended dose remains exceedingly high $(\sim 240 \mathrm{mg} / \mathrm{d})$ due to the significant first-pass effect and the low bioavailability. ${ }^{6}$ Therefore, a safe and bioavailable formulation for NIM is desirable to improve patient compatibility. 
To address the aforementioned problems, various formulations of NIM have been explored, including NIM/ poly(lactic-co-glycolic acid) nanoparticles, NIM/PEG solid dispersion, NIM colloidal dispersion, NIM nanosuspension, and NIM nanocrystal. Although these NIM formulations helped address some of the limitations with Nimotop ${ }^{\circledR}$, they still suffer from low area under the plasma concentrationtime curve $\left(\mathrm{AUC}_{0-t}\right)$, stability issues with crystallization, and complex manufacturing processes. ${ }^{2,4,7-9}$ Self-assembled polymeric micelles represent a novel strategy for improving the solubility and bioavailability of poorly soluble drugs. The surface-active amphiphile Solutol ${ }^{\circledR}$ HS15 (HS15), composed of the PEG-660 ester of 12-hydroxystearic acid, has the potential to increase the solubility of a number of poorly watersoluble compounds such as doxorubicin, 9-nitrocamptothecin, and curcumin. ${ }^{10-12}$ HS15 has been approved as an excipient in a parenteral phytonadione formulation for human use in Canada, ${ }^{13}$ which has been proven to promote solubilization of therapeutics in an aqueous environment, protect therapeutics from degradation without modifying the therapeutic activity, or prevent premature release; eventually, it enhances adhesion and permeation at the biological membranes. HS15 has also been proven to be of low toxicity for oral administration. ${ }^{14}$ However, the detailed transcellular mechanisms of HS15-based micellar systems across the intestinal epithelium remain largely unexplored.

In this study, NIM-loaded HS15 nanomicelles were prepared using a facile one-step self-assembly method. We aimed to improve the oral bioavailability of NIM and the drug's concentration in the brain via oral administration. The dissolution and pharmacokinetic behaviors of NIM nanomicelles were investigated and compared to those of Nimotop ${ }^{\circledR}$. Furthermore, a systemic study has been carried out to elucidate the transport pathways and mechanisms of NIM nanomicelle transport across the intestinal epithelium.

\section{Materials and methods}

\section{Materials and animals}

NIM (99.9\%) was kindly gifted by Baili Pharmaceutical Co, Ltd (Chengdu, People's Republic of China). Solutol ${ }^{\circledR}$ HS15 was a gift from BASF Co, Ltd (Ludwigshafen, Germany). Nimotop ${ }^{\circledR}$ was provided by Bayer AG. Moreover, 1, 1'dioctadecyl-3,3,3',3'-tetramethylindotricarbocyanine iodide (DiR) was purchased from Nanjing KeyGen Biotechnology Co Ltd (Nanjing, People's Republic of China). All other chemicals and solvents were obtained from Sigma-Aldrich (St Louis, MO, USA).
Male Sprague Dawley rats (200-250 g) and Kunming mice (20-25 g) were purchased from Dashuo Biotechnology (Chengdu, People's Republic of China) and maintained under standard housing conditions. All animal experiments were approved by the Ethics Committee of Sichuan University and conducted in accordance with institutional guidelines.

\section{Preparation of nanomicelles}

The NIM-loaded self-assembled nanomicelles were prepared according to the one-step self-assembled technique. Briefly, NIM and HS15 (1:30, w/w) were added in the flask by gently stirring at $60^{\circ} \mathrm{C}$ for 30 minutes. Then, the liquid mixture was hydrated with isotonic saline $(0.9 \% \mathrm{NaCl}[\mathrm{w} / \mathrm{v}], \mathrm{pH} 7.4)$. After stirring for another 10 minutes, nonencapsulated NIM was separated by filtration of the micelle suspensions through a $0.2 \mu \mathrm{m}$ polycarbonate membrane (EMD Millipore, Billerica, MA, USA). The NIM nanomicelles were then obtained. A near-infrared fluorescent probe, DiR, was used and encapsulated in nanomicelles, following the same procedures as for NIM nanomicelles.

\section{Characterization of the nanomicelles}

The particle sizes, polydispersity indexes (PDIs), and zeta potential values of the nanomicelles were measured by dynamic light scattering using Malvern Zetasizer NanoZS90 (Malvern Instruments, Malvern, UK). The size and morphology of the nanomicelles encapsulating NIM were examined using transmission electron microscopy (TEM) (JEM 100CX; JEOL, Tokyo, Japan). The nanomicelles were stained with $1 \%$ uranyl acetate.

The analysis of NIM was performed using a highperformance liquid chromatography (HPLC) system equipped with an ultraviolet detector (Agilent Technologies, Santa Clara, CA, USA). An octadecyl silica (ODS) column (Kromasil, $250 \mathrm{~mm} \times 4.6 \mathrm{~mm}, 5 \mu \mathrm{m}$ ) was used for separation, and the column temperature was $30^{\circ} \mathrm{C}$. The mobile phase consisted of methanol and water $(70 / 30, v / v)$. The flow rate was $1.0 \mathrm{~mL} / \mathrm{min}$ and the detection wavelength was set at $237 \mathrm{~nm}$. All samples were analyzed in triplicate. To estimate the encapsulation efficiency of NIM nanomicelles, the micelle solution was added with tenfold volume of methanol. The solution was properly diluted before HPLC analysis. Encapsulation efficiency (EE) and drug-loading coefficient (DL) were calculated using the following formulas:

$\mathrm{EE} \%=\frac{\text { Weight of NIM measured in nanomicelles }}{\text { Weight of the feeding NIM }} \times 100 \%$ 


$$
\text { DL } \%=\frac{\text { Weight of NIM measured in nanomicelles }}{\left(\begin{array}{c}
\text { Total weight of nanomicelle materials } \\
\text { added }+ \text { weight } \text { of the feeding NIM }
\end{array}\right)} \times 100 \%
$$

\section{Critical micelle concentration}

The critical micelle concentration (CMC) was determined using the pyrene 1:3 ratio method, as previously reported..$^{15}$ The pyrene concentration was set to $6 \times 10^{-7} \mathrm{M}$ for all samples. The polymer concentration varied from $1 \times 10^{-8} \mathrm{mg} / \mathrm{mL}$ to $1 \mathrm{mg} / \mathrm{mL}$. Solutions were stirred for 36 hours before recording the spectrum at room temperature. Fluorescence spectra were recorded using a fluorescence spectrophotometer (Shimadzu, Kyoto, Japan). The excitation wavelength $\left(\lambda_{\mathrm{ex}}\right)$ was $366 \mathrm{~nm}$. The emission wavelengths $\left(\lambda_{\mathrm{em}}\right)$ were those corresponding to the maximum intensities of the first $\left(I_{1}\right.$; $\left.\lambda_{\text {em }}=373 \mathrm{~nm}\right)$ and the third peaks $\left(I_{3} ; \lambda_{\text {em }}=383 \mathrm{~nm}\right)$ in the emission spectrum. To analyze the microenvironment and the CMC derivatives, the ratio $I_{1} / I_{3}$ was determined in triplicate. The emission spectrum and the $I_{1} / I_{3}$ ratio of pyrene depend strongly on the polarity of the environment surrounding the probe molecules. This covers a wide range of polarities from water $\left(I_{1} / I_{3}=1.9\right)$ to cyclohexane $\left(I_{1} / I_{3}=0.6\right)$. This scale was used to evaluate the microenvironment in the micellar core. To determine the $\mathrm{CMC}$, the $I_{1} / I_{3}$ values were averaged over three values plotted versus the polymer concentration. As the polymer concentration in the aqueous solution increased, the $I_{1} / I_{3}$ ratio decreased and then reached a plateau. The CMC was determined at the intersection of the two lines obtained by linear regression.

\section{Stability studies}

To test the long-term stability of the NIM nanomicelles at different temperatures, the nanomicelles were stored at $4^{\circ} \mathrm{C}$, $25^{\circ} \mathrm{C}$, and $40^{\circ} \mathrm{C}$ for 16 weeks. At given time points, the particle size, PDI, zeta potential, and NIM concentration of the NIM nanomicelles were determined in triplicate.

To evaluate the stability in media modeling physiological conditions of the gastrointestinal (GI) tract, NIM nanomicelles were diluted with simulated gastric fluid without pepsin (SGF, pH 1.6) and simulated intestinal fluid without trypsin (SIF, pH 6.5). SGF was composed of $0.2 \% \mathrm{NaCl}, 0.25 \%$ sodium dodecyl sulfate (SDS), and $0.7 \% \mathrm{HCl}$ in water, and the $\mathrm{pH}$ was adjusted to 1.6 by adding concentrated $\mathrm{HCl}$. SIF was composed of $0.3 \%$ dipotassium hydrogen phosphate $\left(\mathrm{K}_{2} \mathrm{HPO}_{4}\right.$ ) with $0.77 \%$ potassium chloride ( $\mathrm{pH}$ adjusted to 6.5 using $\mathrm{NaOH}$ ), $3 \mathrm{mM}$ sodium taurocholate, and $0.75 \mathrm{mM}$ lecithin. NIM nanomicelles were mixed with appropriate volumes of the media (SGF or SIF) to dilute the samples ten times. The diluted samples were incubated at $37^{\circ} \mathrm{C}$. The particle size of the samples was determined at 2 hours, 4 hours, 6 hours, 8 hours, and 12 hours. The experiment was repeated three times.

\section{In vitro drug release}

In vitro NIM release study was carried out according to the reported procedures with modifications ${ }^{16}$ Briefly, $200 \mu \mathrm{L}$ of NIM nanomicelles was introduced into a dialysis membrane bag (molecular weight cutoff $=3.5 \mathrm{kDa}$, Solarbio Science \& Technology Co, Beijing, People's Republic of China), and the end-sealed dialysis bag was incubated in $50 \mathrm{~mL}$ release media at $37^{\circ} \mathrm{C} \pm 0.5^{\circ} \mathrm{C}$ and shaken at a speed of $100 \mathrm{rpm}$. SGF and SIF were used as release media. After 2 hours' incubation of NIM nanomicelles in $50 \mathrm{~mL}$ SGF, the dialysis bags were transferred into $50 \mathrm{~mL}$ SIF for up to 72 hours. Sink conditions were achieved by the addition of SDS in the SGF and lecithin/sodium taurocholate in SIF. At predetermined time intervals, $0.5 \mathrm{~mL}$ of the release media was withdrawn and replaced with an equal volume of the fresh solution (SGF or SIF). The in vitro release behavior of NIM nanomicelles was measured and compared with that of NIM tablets in the suspension from Nimotop ${ }^{\circledR}$. The tablets were pulverized in a mortar. Into each sample, $4.5 \mathrm{~mL}$ of methanol was added and stored until HPLC analysis. All assays were performed in triplicate. Release profiles are presented as cumulative release of NIM in percentage versus time.

\section{Endocytosis pathway}

Cells lines were derived from heterogeneous human epithelial colorectal adenocarcinoma cells, which are commercially available from various cell banks. No ethical approval from the ethics committee of Sichuan University was required for the use of these cell lines. All cell culture studies were performed under laminar flow and sterile conditions. Caco-2 cells were obtained from the Cell Bank of Chinese Academy of Sciences (Shanghai, People's Republic of China) and incubated at $37^{\circ} \mathrm{C}$ with $5 \% \mathrm{CO}_{2}$ under fully humidified conditions. Cells were cultured in Dulbecco's Modified Eagle's Medium (Thermo Fisher Scientific, Waltham, MA, USA) with $10 \%$ fetal bovine serum (FBS; Thermo Fisher Scientific), $2 \mathrm{mM}$ glutamine, 100 units $/ \mathrm{mL}$ penicillin, and $100 \mu \mathrm{g} / \mathrm{mL}$ streptomycin. Before the experiments, Caco-2 cells were seeded into a 12-well cell culture plate (Corning Incorporated, Corning, NY, USA) at $4 \times 10^{4}$ cells per well and cultured for 14 days for the uptake experiments. The culture 
Table I Inhibitors with different functions used in the study of various pathways and their concentrations

\begin{tabular}{|c|c|c|}
\hline Inhibitors & Concentrations & Functions \\
\hline Methyl- $\beta$-cyclodextran (M $\beta C D)$ & $10 \mathrm{mM}$ & $\begin{array}{l}\text { Cholesterol depletion agent; effective inhibitor of lipid raft/caveolae-dependent } \\
\text { endocytosis }^{17}\end{array}$ \\
\hline Amiloride & $20 \mu \mathrm{M}$ & $\begin{array}{l}\mathrm{Na}^{+} / \mathrm{H}^{+} \text {ion channel blocking agent; inhibitor of endocytosis pathway through } \\
\text { macropinocytosis }\end{array}$ \\
\hline Nystatin & $30 \mu \mathrm{M}$ & Inhibitor of lipid/caveolae-dependent endocytosis by cholesterol sequestration effect ${ }^{17}$ \\
\hline Chlorpromazine & $10 \mu g / m L$ & $\begin{array}{l}\text { Inhibitor of clathrin-mediated endocytosis by interacting with vicinal dithiol-containing } \\
\text { molecules }^{19}\end{array}$ \\
\hline Filipin & $6 \mu \mathrm{g} / \mathrm{mL}$ & Inhibitor of lipid/caveolae-mediated endocytosis ${ }^{20}$ \\
\hline Indomethacin & $6 \mu \mathrm{g} / \mathrm{mL}$ & Common inhibitor of cyclooxygenase $(\mathrm{COX})^{21}$ \\
\hline LY294002 & $\mathrm{I} \mu \mathrm{M}$ & Inhibitor of phosphatidylinositol 3-kinase $(\mathrm{PI} 3 \mathrm{~K})^{22}$ \\
\hline
\end{tabular}

medium was replaced every 2 days for the first week, and every day thereafter, to form cell monolayers.

The endocytosis pathway of NIM nanomicelles was investigated by the addition of specific pharmacological inhibitors for specific cellular uptake pathways, as described in Table 1. Before the addition of NIM nanomicelles, Caco-2 cells grown on 12-well plate were preincubated with inhibitors for 1 hour at $37^{\circ} \mathrm{C}$. Following addition of $5 \mu \mathrm{M}$ NIM, nanomicelles were incubated with cells for a further 1 hour. During the nanomicelle incubation, the concentration of inhibitors was maintained as constant. After the coincubation of nanomicelles and inhibitors, cellular uptake was aborted using cold Hank's buffered salt solution (HBSS; $137.93 \mathrm{mM} \mathrm{NaCl}, 5.33 \mathrm{mM} \mathrm{KCl}$, $4.17 \mathrm{mM} \mathrm{NaHCO}_{3}, 0.44 \mathrm{mM} \mathrm{KH} \mathrm{PO}_{4}, 0.34 \mathrm{mM} \mathrm{Na} \mathrm{HPO}_{4}$, and $5.56 \mathrm{mM}$ D-glucose). The cells obtained after nanomicelle incubation were digested, centrifuged, rinsed using HBBS, and then lysed using freeze-thaw method. The final cell lysis solution was first tested using the bicinchoninic acid assay (Applygen, Beijing, People's Republic of China) to obtain the total protein content. The remaining lysis solution was then blended with methanol to dissolve the NIM present in nanomicelles and examined using HPLC, as described earlier.

The temperature dependency of endocytosis of NIM nanomicelles was detected by HPLC. Caco-2 cells in 12-well plate were incubated with $5 \mu \mathrm{M}$ NIM nanomicelles at $37^{\circ} \mathrm{C}$ and $4^{\circ} \mathrm{C}$, respectively. At determined time points, cells from plate wells were digested and then lysed using the method described earlier. Obtained samples were subjected to HPLC to obtain the amount of NIM.

In addition, the effect of NIM nanomicelles on F-actin filaments in Caco-2 cell monolayer was evaluated under laser confocal scanning microscopy (LCSM) (Fluoview ${ }^{\mathrm{TM}} 1000$; Olympus Corporation, Tokyo, Japan). Briefly, Caco-2 cells were grown on round glass coverslips $(10 \mathrm{~mm})$ in 24 -well culture plates (Corning Incorporated) and cultured as described earlier. NIM nanomicelles were added to the freshly replaced FBS-free Dulbecco's Modified Eagle's Medium (5 $\mu \mathrm{M} / \mathrm{mL}$ of NIM) and incubated with the cell monolayer for the measurement. At predetermined time intervals, the cell monolayer was rinsed with cold phosphate-buffered saline (pH 7.4), fixed with 4\% paraformaldehyde solution, and stained with fluorescein isothiocyanate (FITC)-phalloidin for imaging under LCMS. The cell monolayer without any treatment was used as negative control.

\section{Exocytosis of NIM nanomicelles in Caco-2 cell monolayers}

Caco-2 cells were seeded in 12-well plates and cultured as described earlier. Before investigation of the exocytosis mechanism, Caco- 2 cells were first incubated with $5 \mu \mathrm{M}$ NIM nanomicelles for 1 hour at $37^{\circ} \mathrm{C}$. Then, nanomicelle dispersion was replaced by HBSS, methyl- $\beta$-cyclodextrin $(\mathrm{M} \beta \mathrm{CD}$, $10 \mathrm{mM}), \mathrm{LY} 294002(1 \mu \mathrm{M})$, verapamil $(100 \mu \mathrm{g} / \mathrm{mL})$, EXO1 $(1 \mu \mathrm{M})$, and indomethacin $(300 \mu \mathrm{M})$, respectively, at $37^{\circ} \mathrm{C}$ for subsequent reincubation. To inhibit energy-dependent exocytosis, cell monolayers were reincubated at $4^{\circ} \mathrm{C}$. The intracellular NIM was determined by HPLC as described herein.

\section{Transcellular transport of NIM nanomicelles across Caco- 2 cell monolayer}

Before the experiments, Caco- 2 cells were seeded onto polycarbonate membrane filters $\left(0.4 \mu \mathrm{m}\right.$ pore size, $1.12 \mathrm{~cm}^{2}$ growth area) in Transwell ${ }^{\circledR}$ cell culture chambers (Corning Costar, Cambridge, MA, USA) at a density of $1 \times 10^{5}$ cells per insert. The culture medium $(0.5 \mathrm{~mL}$ per insert and $1.5 \mathrm{~mL}$ per well) was replaced every 2 days in the first 2 weeks, but changed every day thereafter. After 21 days' culture, cell monolayers were used for the following assays. To investigate the influence of nanomicelles and Nimotop ${ }^{\circledR}$ on the adsorption properties of NIM, Caco-2 cells were used as an in vitro model of the GI tract epithelium. Before the experiments, cell monolayers were washed twice with 
HBSS at $37^{\circ} \mathrm{C}$ for 15 minutes. The transepithelial electrical resistance (TEER) of the monolayer was measured using a Millicell ${ }^{\circledR}$-ERS system (EMD Millipore) to determine both the formation of the monolayer and its integrity during the experiment. Only the cell monolayers with TEER values $>700 \Omega \cdot \mathrm{cm}^{2}$ were used.

To investigate transport behaviors, NIM nanomicelles and Nimotop ${ }^{\circledR}$ were diluted with HBSS to a final concentration of 5.0 $\mu \mathrm{M}$ NIM. The drugs were applied to Caco-2 cell monolayers from the apical to the basolateral direction by adding $0.5 \mathrm{~mL}$ of a test solution at the apical side and adding $1.5 \mathrm{~mL}$ of HBSS at the basolateral side. A volume of $500 \mu \mathrm{L}$ of the sample was taken from the basolateral side at predetermined time points (15 minutes, 30 minutes, 60 minutes, 90 minutes, 120 minutes, 150 minutes, and 180 minutes) and replaced with the same volume of fresh buffer immediately. Samples were mixed with $2.5 \mathrm{~mL}$ methanol and shaken for 1 minute using a vortex mixer and centrifuged at 10,000 rpm for 10 minutes. The supernatant was filtered using a $0.2 \mu \mathrm{m}$ membrane filter. Samples were analyzed by liquid chromatography-tandem mass spectrometry (LC-MS/ MS) (Agilent Technologies) and the concentrations of NIM were calculated using a standard calibration curve. The LC-MS/MS system consisted of an Agilent 1200 series pumping system, including an SL binary pump, degasser, autosampler, and a triple quadrupole mass spectrometer. A Diamonsil ${ }^{\circledR}$ ODS column $(1.8 \mu \mathrm{m}, 50 \times 4.6 \mathrm{~mm})$ with a corresponding guard column (ODS, $5 \mu \mathrm{m}$ ) was used for separation and maintained at $30^{\circ} \mathrm{C}$. The mobile phase consisted of $30 \%$ deionized water and $70 \%$ methanol (v/v) at a flow rate of $0.4 \mathrm{~mL} / \mathrm{min}$. MS operating conditions were established to detect NIM. MS was carried out under negative electrospray ionization and multiple reaction monitoring mode. Nitrogen was used as the nebulizer gas. The gas flow rate was $10 \mathrm{~mL} / \mathrm{min}\left(350^{\circ} \mathrm{C}\right)$ and the pressure was set at $35 \mathrm{psi}$. Fragmentor voltages were $138 \mathrm{eV}$ and collision energies were $16 \mathrm{eV}$. Ion reaction used the parent ion at $\mathrm{m} / \mathrm{z} 417.2$ and the daughter ion at $m / z$ 121.9.

To directly observe whether NIM nanomicelles are transported in the intact form across the monolayer, the cell monolayer was incubated with nanomicelles at $37^{\circ} \mathrm{C}$ for 1 hour. The basolateral medium was collected and observed under TEM.

\section{Absorption of NIM nanomicelles in the intestine}

The absorption of NIN nanomicelles compared with that of Nimotop ${ }^{\circledR}$ was measured in mice. Briefly, NIM nanomicelles and Nimotop ${ }^{\circledR}$ were administered to the fasted mice at $60 \mathrm{mg} / \mathrm{kg}$ of NIM by oral gavage. At 0.5 hour, 1.0 hour, and 4.0 hours after oral administration, mice were sacrificed, and different intestinal segments of duodenum, jejunum, and ileum were removed. The selected tissues were carefully rinsed with cold saline, accurately weighted, and homogenized with $0.5 \mathrm{~mL}$ acetonitrile on the Precellys 24 lysis instrument (Bertin, Montigny-le-Bretonneux, France). The tissue homogenates were centrifuged at 14,000 rpm for 10 minutes (Thermo Scientific Heraeus Biofuge Stratos, Osterode, Germany) and the supernatant was analyzed by LC-MS/MS method as described herein. The NIM concentration in each tissue was normalized by the weight of the selected tissue.

To visualize distributions of nanomicelles in the intestine, DiR-loaded nanomicelles were administered to mice at $4 \mathrm{mg} / \mathrm{kg}$ of DiR by oral gavage. At 0.5 hour, 1.0 hour, and 4.0 hours after oral administration, mice were sacrificed and the intestines were removed. These tissues were carefully rinsed and scanned using a multimodal imaging system (IVIS Spectrum; Caliper, Alameda, CA, USA) with an excitation bandpass filter at $730 \mathrm{~nm}$ and an emission at $790 \mathrm{~nm}$. Exposure time was set at 30 seconds. The near-infrared fluorescence signal intensities were measured.

\section{Pharmacokinetic and biodistribution study}

Before the experiments, rats that fasted overnight with free access to water were randomly divided into two groups. NIM nanomicelles and Nimotop ${ }^{\circledR}$ were given to rats at $60 \mathrm{mg} / \mathrm{kg}$ of NIM by oral gavage. Blood samples were collected at predetermined time points, immediately centrifuged at 6,000 rpm for 5 minutes, and stored at $-80^{\circ} \mathrm{C}$ before analysis. NIM was extracted from the plasma with acetonitrile and determined by LC-MS/MS. The pharmacokinetic parameters were calculated using the noncompartmental model, and the relative oral bioavailability was calculated as the $\mathrm{AUC}_{0-t}$ value of NIM nanomicelles compared to that of Nimotop $^{\circledR}$.

The biodistribution of orally administered NIM nanomicelles was measured and compared with that of Nimotop ${ }^{\circledR}$. Mice were randomly divided into two groups $(n=3)$ and administered NIM nanomicelles and Nimotop ${ }^{\circledR}(60 \mathrm{mg} / \mathrm{kg})$ by oral gavage. At 1.0 hour after oral administration, mice were sacrificed and various organs, including heart, liver, spleen, lung, kidney, and brain, were removed. These tissues were rinsed with cold saline, accurately weighted, and homogenized with $0.5 \mathrm{~mL}$ of acetonitrile on the Precellys 24 lysis instrument. The tissue homogenates were centrifuged at $14,000 \mathrm{rpm}$ for 10 minutes. The NIM concentration in the supernatant was determined, and the biodistribution of 
NIM in each organ was normalized by the weight of the selected tissues.

\section{Statistical analysis}

Statistical analysis was performed by two-tailed Student's $t$-test. The differences between two groups were considered statistically different for $P<0.05$ and significantly different for $P<0.01$.

\section{Results and discussion \\ Characterization of NIM nanomicelles}

HS15 has been demonstrated to enhance the oral bioavailability of various lipophilic drugs. ${ }^{13}$ HS15-based NIM nanomicelles were prepared by a one-step self-assembly method (Figure 1A). The method can be easily scaled up without using any organic solvent. NIM nanomicelles displayed uniform near-spherical-shaped particles with
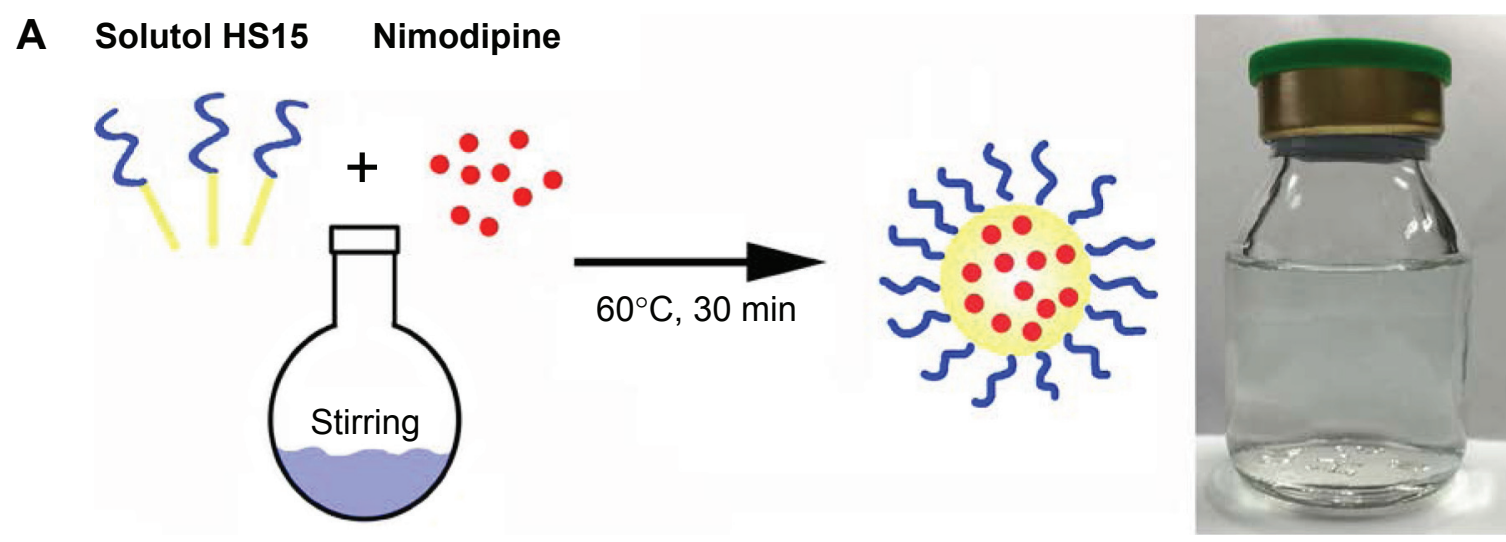

B
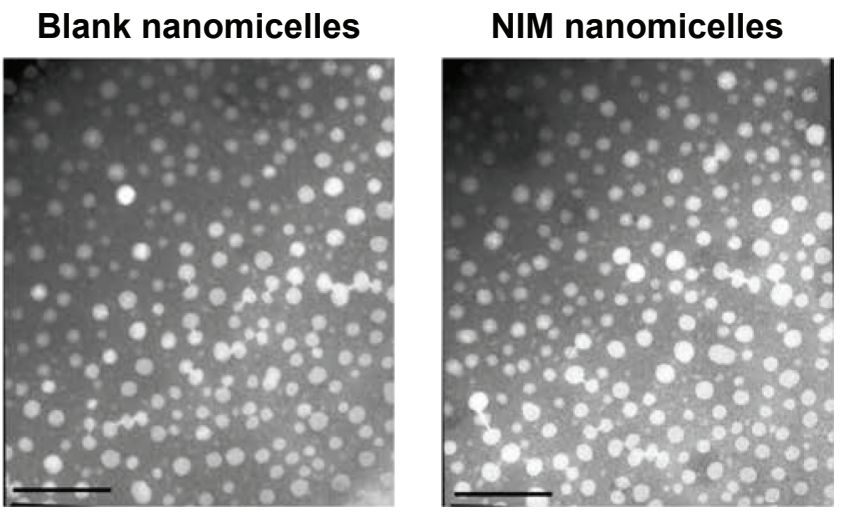

C

Size distribution by intensity

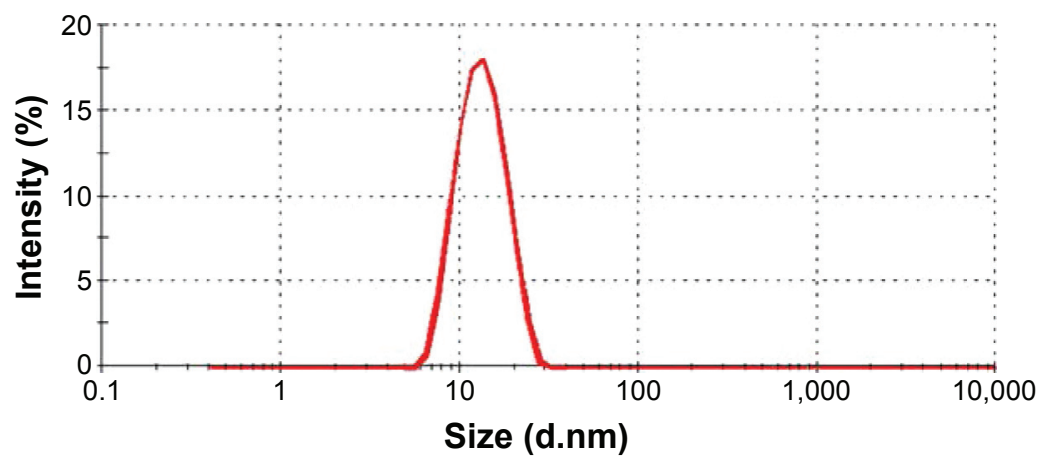

Figure I Preparation and characterization of NIM nanomicelles.

Notes: (A) Scheme of the preparation of NIM nanomicelles; (B) typical TEM photo of NIM nanomicelles, scale bar =100 nm; magnification was I00,000×; (C) size distribution of NIM nanomicelles by intensity with oversize curve.

Abbreviations: HSI5, PEG-660 ester of 12-hydroxystearic acid; NIM, nimodipine; PEG, polyethylene glycol; TEM, transmission electron microscopy; min, minutes; d, diameter. 

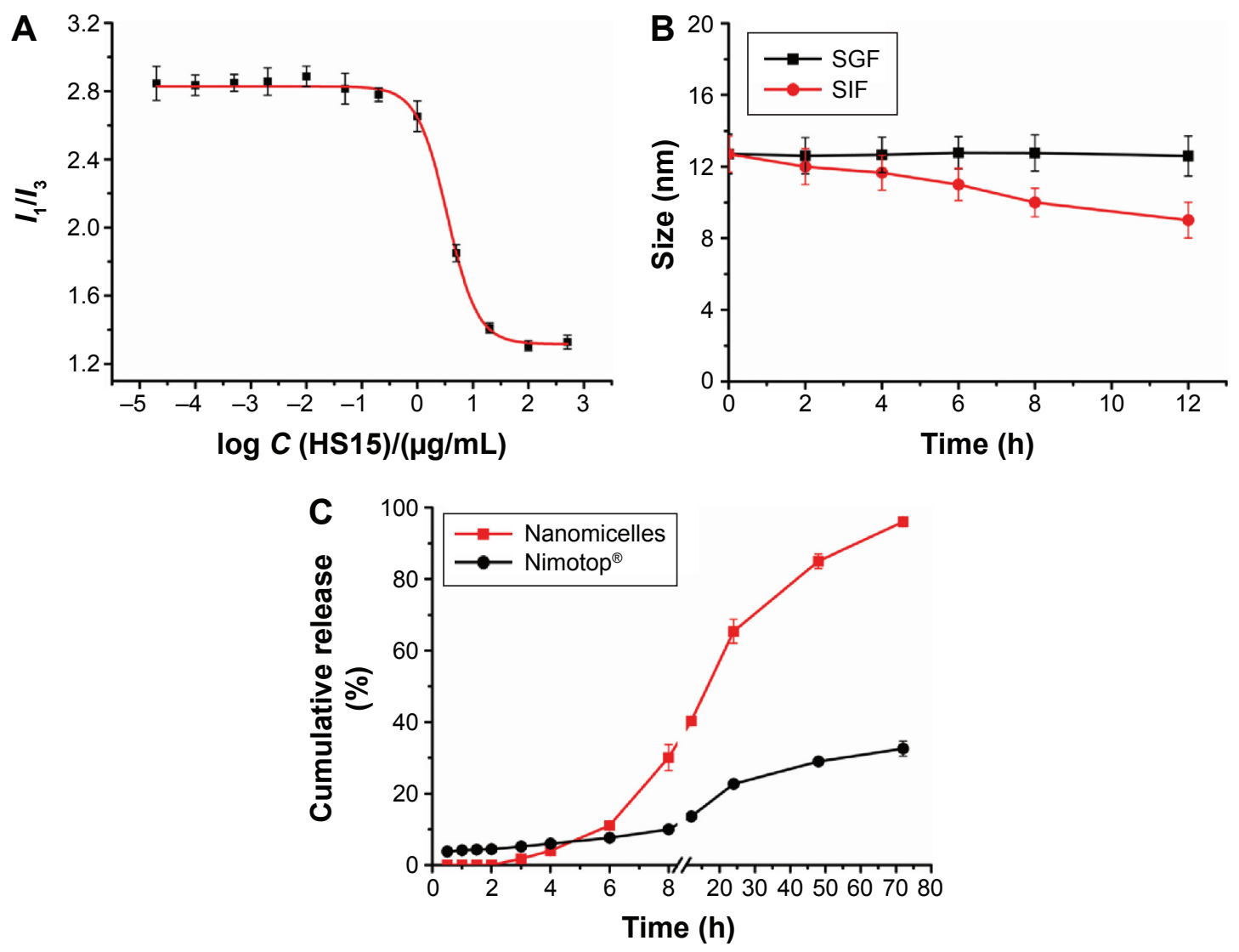

Figure 2 In vitro evaluation of NIM nanomicelles.

Notes: (A) $I_{1} / I_{3}$ ratio versus polymer concentration of HSI5 (log $C$ ) in water. (B) Stability of NIM nanomicelles in SGF (pH I.6) and SIF (pH 6.5). (C) In vitro release profiles of NIM nanomicelles and Nimotop ${ }^{\circledR}$ in different release media. Data represent mean \pm SD $(n=3)$.

Abbreviations: HSI5, PEG-660 ester of I2-hydroxystearic acid; I III ratio, pyrene I:3 peak intensity ratio; log $C$, logarithms of the concentration of HSI5; NIM, nimodipine; PEG, polyethylene glycol; SD, standard deviation; SGF, simulated gastric fluid without pepsin; SIF, simulated intestinal fluid without trypsin; h, hours.

an average size distribution of $\sim 12.57 \pm 0.21 \mathrm{~nm}$ (PDI $=0.071 \pm 0.011$ ) (Figure $1 \mathrm{~B}$ and $\mathrm{C}$ ). The encapsulation efficiency and drug loading of NIM in nanomicelles were $95.75 \% \pm 12.93 \%$ and $3.23 \% \pm 0.48 \%$, respectively. NIM nanomicelles were negatively charged, with a zeta potential value of $-8.56 \pm 1.19 \mathrm{mV}$.

\section{Critical micelle concentration}

The CMC of HS15 was determined by the pyrene 1:3 ratio method. ${ }^{15}$ When the concentration of HS15 was increased, shifts occurred in the emission spectra $\left(I_{1}: \lambda_{\text {em }}\right.$ from $372 \mathrm{~nm}$ to $374 \mathrm{~nm}$; and $I_{3}: \lambda_{\text {em }}$ from $383 \mathrm{~nm}$ to $385 \mathrm{~nm}$ ) of pyrene, resulting from a shift from an aqueous polar environment to a more hydrophobic medium. The CMC of HS15 was determined as $3.45 \pm 1.01 \mu \mathrm{g} / \mathrm{mL}$ (Figure 2A). As expected, the micelle core was very hydrophobic $\left(I_{1} / I_{3} \approx 2.07\right)$. With the relatively small CMC value, HS15-based micelles are expected to withstand the rapid dilution by gastric fluid in vivo and maintain the stability of the encapsulated drugs after oral administration.

\section{Stability of NIM nanomicelles}

A major problem with micelle formulations is their long-term stability. To investigate the stability profiles of NIM-loaded nanomicelles over time, the hydrodynamic diameters, zeta potentials, and the percentage of NIM within micelles were characterized, which were shown to slightly increase over time. Importantly, the results revealed that the hydrodynamic diameters of micelles at different temperatures $\left(4^{\circ} \mathrm{C}, 25^{\circ} \mathrm{C}\right.$, and $40^{\circ} \mathrm{C}$ ) all remained $<50 \mathrm{~nm}$, while the percentage of NIM encapsulated within micelles remained above $90 \%$ for up to 4 months, indicating that NIM micelles are a thermodynamically stable system (Table 2 ).

To ensure the delivery of the carried drug to its site of absorption, micelles must be able to resist rapid dissociation upon dilution and exposure to the harsh conditions of the GI tract. Thus, the stability of NIM nanomicelles was investigated at $37^{\circ} \mathrm{C}$ in $\mathrm{SGF}(\mathrm{pH} 1.6)$ or SIF ( $\mathrm{pH}$ 6.5) for 12 hours. The size of micelles remained unchanged for up to 12 hours in SGF. In contrast, the size of micelles slightly decreased from $12.71 \mathrm{~nm}$ to $9.01 \mathrm{~nm}$ over 12 hours in SIF 
Table 2 Stability of NIM nanomicelles at different temperature

\begin{tabular}{lllll}
\hline Temperature & Average size $(\mathbf{n m})$ & PDI & Zeta potential $(\mathbf{m V})$ & Content $(\%)$ \\
\hline Control & $12.57 \pm 0.21$ & $0.071 \pm 0.011$ & $-8.56 \pm 1.19$ & 100 \\
$4^{\circ} \mathrm{C}$ & $12.74 \pm 0.75$ & $0.101 \pm 0.017$ & $-8.10 \pm 1.10$ & $99.99 \pm 0.91$ \\
$25^{\circ} \mathrm{C}$ & $12.71 \pm 0.86$ & $0.078 \pm 0.016$ & $-8.18 \pm 1.26$ & $99.90 \pm 1.03$ \\
$40^{\circ} \mathrm{C}$ & $12.90 \pm 0.91$ & $0.090 \pm 0.011$ & $-9.02 \pm 1.62$ & $99.71 \pm 1.12$ \\
\hline
\end{tabular}

Note: Data presented as mean \pm standard deviation.

Abbreviations: NIM, nimodipine; PDI, polydispersity index.

(Figure 2B). Because the retention time of nanomicelles in the GI tract is $<6$ hours, the NIM nanomicelles were expected to maintain their structural stability during the course of absorption in the GI tract.

\section{In vitro release of NIM}

To simulate the in vivo biological environment, the in vitro release profiles of NIM were characterized under a $\mathrm{pH}$ gradient simulating the $\mathrm{pH}$ evolution of the GI tract (SGF, pH 1.6; SIF, pH 6.6). The cumulative release percentage of NIM from nanomicelles was $\sim 65.06 \% \pm 3.33 \%$ in SGF/ SIF within 24 hours, while Nimotop ${ }^{\circledR}$ showed a cumulative release of $<30 \%$ in 24 hours. As shown in Figure 2C, the cumulative release of NIM in SIF was much higher than that in SGF solution, which might be related to the different $\mathrm{pH}$ values. As reported previously, ${ }^{16}$ the swelling and erosion of the polymers could be inhibited at lower $\mathrm{pH}$, thus decreasing cumulative drug release.

To elucidate the release mechanism of NIM, the release kinetics of NIM was further fitted to several mathematical models (Table 3). For the regression of NIM release from NIM nanomicelles, the zero-order equation provided the best regression results, followed by the Weibull equation. The empirical Peppas model was used to gain further insight into the release mechanisms. According to the Peppas model, $0.43<\mathrm{b}<0.85$ indicates an anomalous transport behavior from spheres, ie, a superposition of both Fickian diffusion and Case II transport. Regarding NIM nanomicelles, the release kinetics of NIM could be interpreted as both Fickian diffusion and Case II transport driven.
The SGF/SIF setup successfully produced a sink condition without interfering with the micelle structure, which could be concluded from stability and in vitro release experiments. However, the physiological conditions cannot be perfectly mimicked by any simulated fluid. Whether or not the orally administrated micelles are affected by constituents of the fluid in the GI tract remains unknown. Thus, the oral bioavailability of NIM micelles should be further explored using in vivo experiments.

\section{Endocytosis of NIM nanomicelles by Caco-2 cells}

The transcellular transport of nanomedicines through epithelial cells may involve various processes, such as cell surface binding, endocytosis, intracellular trafficking, exocytosis, and transcytosis, and most of them relate to the subcellular structures in cells. ${ }^{23}$ Nanocarriers may engage in transport in cells via different pathways, which may be specific or nonspecific, energy dependent or independent. ${ }^{24}$ As a result, the transport mechanisms of nanomedicines across epithelial cells remain to be explored. In the following study, we investigated the effect of various endocytosis inhibitors on the cellular uptake of NIM nanomicelles (Figure 3A) in Caco-2 cell monolayers. All inhibitors demonstrated different levels of suppression on the internalization of NIM nanomicelles in Caco-2 cells. Among them, $\mathrm{M} \beta \mathrm{CD}$ triggered the most significant uptake reduction $(53.1 \%)$, followed by chlorpromazine (32.7\%), filipin (26.2\%), LY294002 (25.3\%), nystatin (23.8\%), indomethacin (18.1\%), and amiloride (17.6\%). The functions of each inhibitor used herein are summarized in Table 1.

Table 3 Regression parameters of NIM release behaviors from NIM nanomicelles in different media via mathematical modeling

\begin{tabular}{lllll}
\hline Function & A & B & C & NA \\
\hline Zero order: $y=\mathrm{a} \cdot t$ & $1.66 \pm 0.19$ & NA & NA & 0.88845 \\
First order: $y=\mathrm{a} \cdot[1-\exp (-\mathrm{b} \cdot t)]$ & $96.88 \pm 3.80$ & $0.05 \pm 0.01$ & NA & 0.99027 \\
Higuchi: $y=\mathrm{a} \cdot t^{0.5}$ & $11.97 \pm 0.66$ & $\mathrm{NA}$ & $\mathrm{NA}$ & 0.94843 \\
Korsmeyer-Peppas: $y=\mathrm{a} \cdot t^{\mathrm{b}}$ & $10.05 \pm 2.66$ & $0.54 \pm 0.07$ & $1.04 \pm 0.11$ & 0.94529 \\
Weibull: $y=\mathrm{a} \cdot\left[1-\exp \left(-\mathrm{b} \cdot t^{\mathrm{c}}\right)\right]$ & $95.56 \pm 4.99$ & $0.04 \pm 0.01$ & & 0.98857 \\
\hline
\end{tabular}

Note: Data presented as mean $\pm S D(n=3)$.

Abbreviations: NA, not available; NIM, nimodipine; SD, standard deviation. 
A

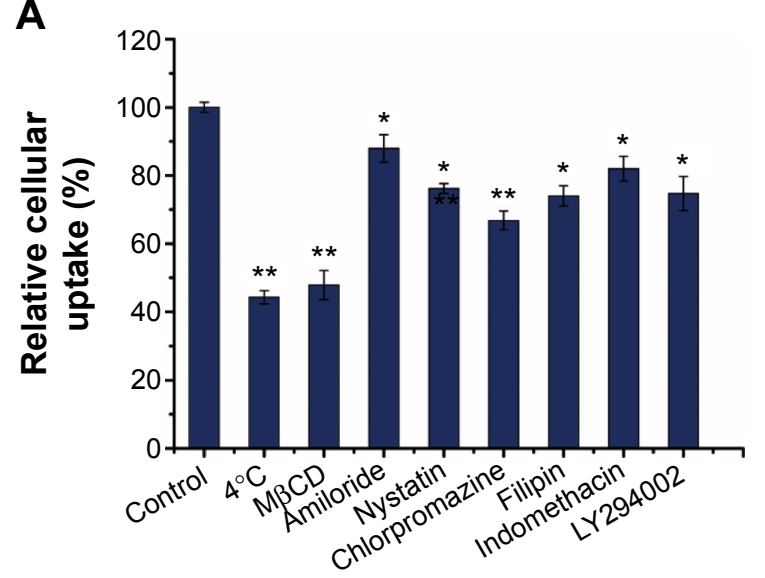

C

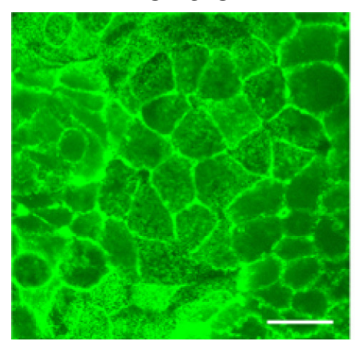

B

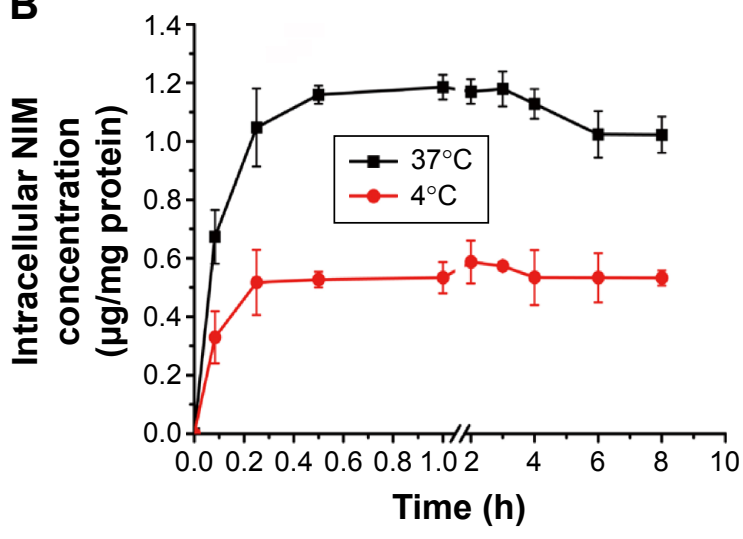

$10 \min$

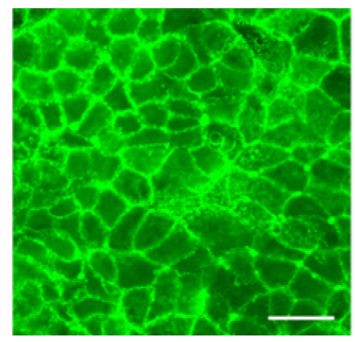

$30 \min$

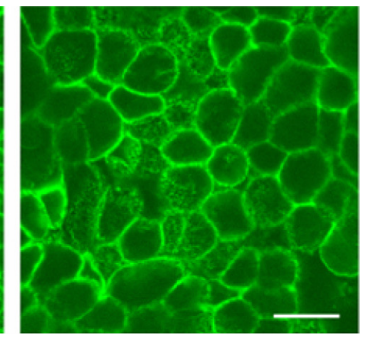

Figure 3 Endocytosis mechanism of NIM nanomicelles in Caco-2.

Notes: (A) Cellular uptake under different conditions; (B) quantitative detection of intracellular NIM during endocytosis process at $37^{\circ} \mathrm{C}$ and $4^{\circ} \mathrm{C}$, respectively; (C) effect of the nanomicelles on F-actin filaments of Caco- 2 cell monolayer observed under LCSM, scale bar $=25 \mu \mathrm{m}$. Magnification was $400 \times$. Data represent mean \pm SD ( $=3$ ). $* P<0.05$, versus control; $* * P<0.01$, versus control.

Abbreviations: HSI5, PEG-660 ester of I2-hydroxystearic acid; NIM, nimodipine; PEG, polyethylene glycol; LCSM, laser confocal scanning microscopy; M $\beta C D$, methyl- $\beta$ cyclodextrin; SD, standard deviation; h, hours; min, minutes.

Clathrin-mediated endocytosis is a common pathway involved in the internalization of multiple extracellular macromolecules and nanoparticles. Here, chlorpromazine, a specific inhibitor of clathrin-mediated endocytosis, ${ }^{19}$ significantly reduced the internalization of NIM nanomicelles, suggesting the involvement of clathrin-mediated endocytosis. In addition, the internalization of extracellular substances can also be regulated by cholesterol-rich microdomains known as lipid rafts and caveolae. ${ }^{25}$ As inhibitors of the lipid raft/caveolae pathway, ${ }^{17}$ $\mathrm{M} \beta C D$ and nystatin also showed an obvious inhibitory effect on the internalization of NIM nanomicelles (Figure 3A), indicating that the lipid raft/caveolae pathway was probably involved. Additionally, extracellular macromolecules can be internalized via macropinocytosis by ruffling the plasma membrane to form cargo-loaded macropinosomes. ${ }^{26}$ Here, amiloride ${ }^{18}$ has been found to decrease the cellular uptake of NIM nanomicelles in Caco- 2 cells, revealing the existence of this pathway. The inhibitory effect of filipin on the cellular uptake of NIM nanomicelles further verified the lipid raft/ caveolae-mediated endocytosis. As a common inhibitor of cyclooxygenase $(\mathrm{COX})$, indomethacin catalyzes the oxygenation of arachidonic acid (AA) to form prostaglandin $G_{2}$
$\left(\mathrm{PGG}_{2}\right)$, as well as the metabolism of $\mathrm{PGG}_{2}$ to $\mathrm{PGH}_{2} \cdot{ }^{27} \mathrm{AA}$ and its metabolites thus play a critical role in the apical endocytosis of MDCK cells. ${ }^{27}$ Here, indomethacin was proven to significantly decrease the internalization of NIM nanomicelles by the COX- and AA-mediated pathway. As previously reported, ${ }^{21}$ COX-2 is found to co-localize with caveolin-1 and reversely regulates the caveolae-mediated endocytosis. Thus, COX might function in the lipid raft/caveolae-dependent internalization of NIM nanomicelles. In sum, the internalization of NIM nanomicelles was probably regulated by multiple mechanisms, further indicating the complexity of potential interactions between nanomaterials and the biological system.

Phosphatidylinositol 3-kinases (PI3Ks) phosphorylate phosphatidylinositol and its derivatives generate important secondary messengers that are important regulators of cell metabolism, cytoskeletal remodeling, and membrane trafficking. ${ }^{28}$ A recent study ${ }^{22}$ showed that regulated synthesis and turnover of phosphoinositides by membraneassociated phosphoinositide kinases and phosphatases spatially restrict the location of effectors critical for cellular transport processes, such as clathrin-mediated endocytosis, autophagy, phagocytosis, macropinocytosis, and 


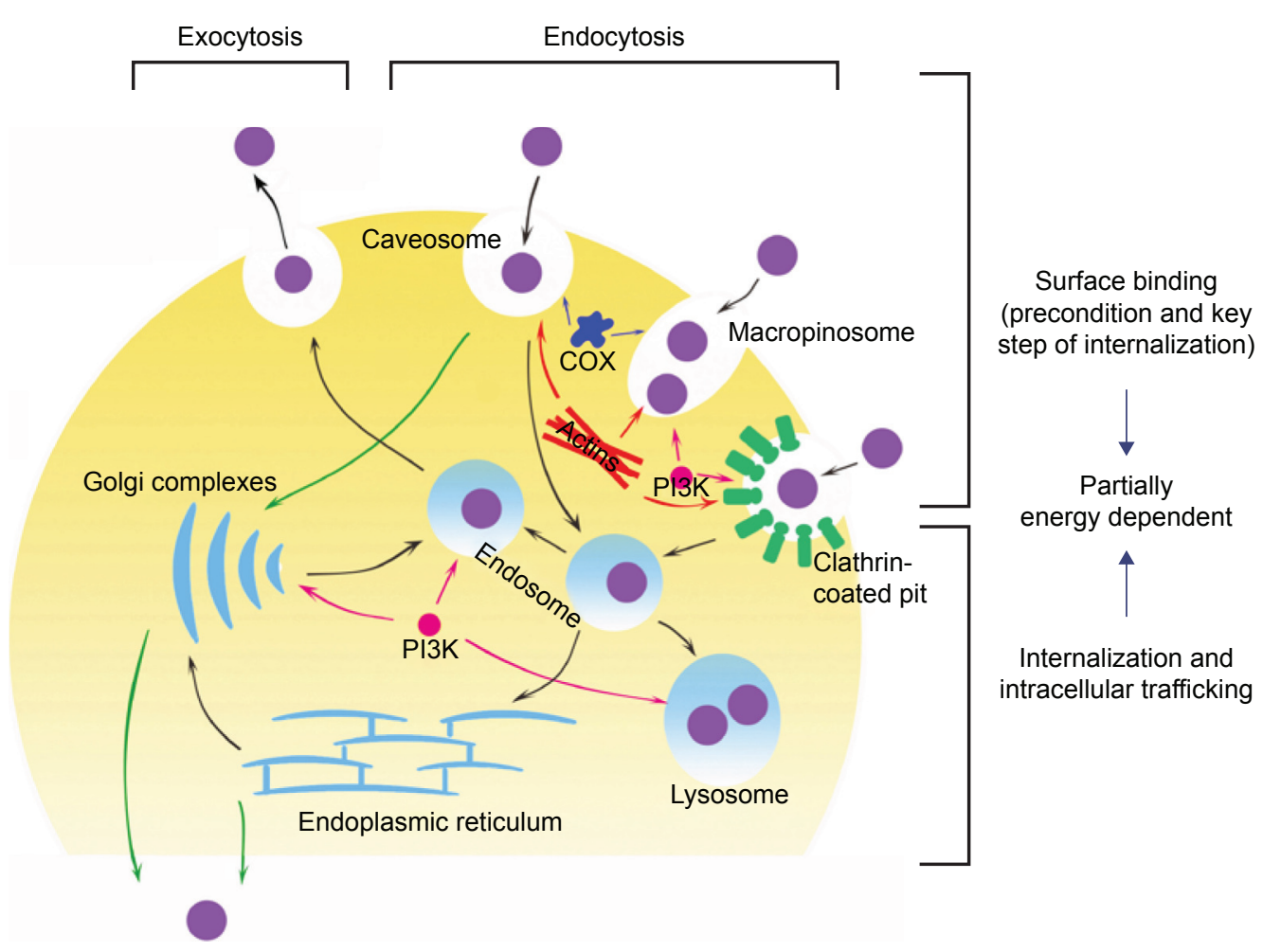

Figure 4 Schematic diagram of the nonspecific and partially energy-dependent transport of polymer nanoparticles in Caco-2 epithelial cells.

Notes: Black arrows represent the pathways we have demonstrated in this study, and green arrows indicate the proven pathways in previous reports. Red, blue, and pink arrows represent the proven regulation by actin, COX, and PI3K, respectively.

Abbreviations: COX, cyclooxygenase; PI3K, phosphatidylinositol 3-kinase.

biosynthetic trafficking. LY294002, a PI3K inhibitor, significantly decreased the intracellular uptake of NIM nanomicelles, which demonstrated that the PI3K pathway played a critical role in the endocytosis of NIM nanomicelles.

Next, we compared the cellular uptake of NIM nanomicelles at $37^{\circ} \mathrm{C}$ and $4^{\circ} \mathrm{C}$ (Figure 3B). The intracellular concentration of NIM at $37^{\circ} \mathrm{C}$ was significantly higher than that at $4^{\circ} \mathrm{C}$. Temperature has been found to significantly influence the ligand-receptor interaction, the internalization of ligand-receptor complexes, and the bioactivities of multiple membrane proteins including F-actins. ${ }^{29}$ As a result, temperature dependency is usually regarded as an indicator of energy-dependent active trafficking of macromolecules. Our results confirmed that the internalization of NIM nanomicelles is an active and energy-dependent endocytic pathway, which is summarized in Figure 4.

To gain insight into the morphology of Caco-2 cell monolayers, cells were stained with FITC-phalloidin. As shown in Figure 3C, after 10 minutes' treatment of NIM nanomicelles, the fluorescence intensity was not significantly reduced compared to that of the negative control. After 30 minutes of incubation, the actin filaments were obviously disrupted, with intermittent fluorescent lumping encircling the cells with reduced fluorescence intensity. The role of actins in the endocytic trafficking of extracellular macromolecules in polarized epithelial cells has been previously clarified. ${ }^{30}$ As an important cytoskeleton component connecting multiple organelles and endosome vesicles, actins participate in multiple internalization processes and effectively regulate the intracellular location of cargos. Thus, the co-localization and intracellular distribution analysis showed that actins were involved in the internalization of NIM nanomicelles by Caco- 2 cells.

\section{Exocytosis mechanism in Caco-2 cell monolayer}

Efflux transporters on the apical membrane of the enterocytes, which belong to the ATP-binding cassette (ABC) family, excrete their substrates from the cells into the intestinal lumen, thereby lowering the intracellular concentration and decreasing intestinal absorption. ${ }^{31}$ The three major types of $\mathrm{ABC}$ transporters expressed on the apical side of the epithelial cells include multidrug resistance protein, multidrug resistance-associated protein 2 , and breast cancer resistance protein, which pump out their substrates, including drugs, back into the gut lumen, thus forming a protective barrier in the intestine. Among these transporters, P-glycoprotein (P-gp) 


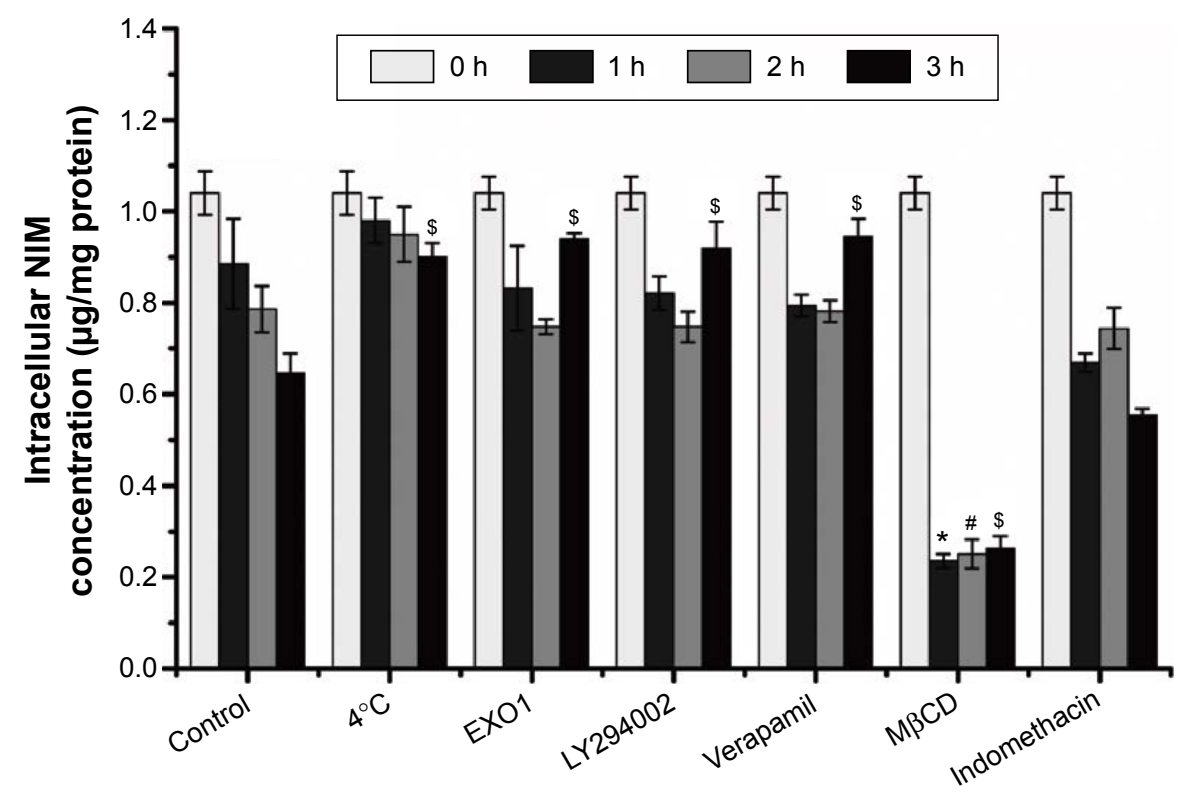

Figure 5 The influence of different inhibitors on intracellular NIM nanomicelles during the reincubation.

Notes: Data represent mean \pm SD $(n=3)$. $* P<0.05$, versus control at I hour. ${ }^{\sharp} P<0.05$, versus control at 2 hours. ${ }^{\$} P<0.05$, versus control at 3 hours.

Abbreviations: HSI5, PEG-660 ester of I2-hydroxystearic acid; NIM, nimodipine; PEG, polyethylene glycol; M $\beta C D$, methyl- $\beta$-cyclodextrin; SD, standard deviation; h, hours.

plays a key role due to its broad substrate specificity and high expression level in the intestinal epithelium. To explore the effect of P-gp on the exocytosis of NIM nanomicelles, the activity of P-gp was inhibited by verapamil, which resulted in the decreased exocytosis of NIM at 3 hours. PI3K regulates the transport between the Golgi apparatus and vacuole/ lysosome. ${ }^{28}$ LY294002 was proven to inhibit the Golgi/ lysosome pathway in the exocytosis of NIM nanomicelles at 3 hours. Both Golgi complex and endoplasmic reticulum (ER) are important regulators for secretory ER/Golgi complex pathway and the endocytic recycling pathway. ${ }^{32} \mathrm{Here}, \mathrm{EXO} 1$ was shown to inhibit the exocytosis of NIM nanomicelles at 3 hours, indicating that the ER/Golgi pathway was involved in the exocytosis of NIM nanomicelles. ${ }^{33}$ On the basis of Figure 5, it seemed that verapamil, EXO1, and LY294002 functioned more significantly at 3 hours than in the early period. This can be explained by the fact that many inhibitors have time-dependent behavior. ${ }^{34}$ The addition of $\mathrm{M} \beta \mathrm{CD}$ as a cholesterol depletion agent triggered a dramatic reduction of intracellular NIM concentration (Figure 5). Thus, the presence of $\mathrm{M} \beta \mathrm{CD}$ greatly enhanced the exocytosis, probably via a lipid raft/caveolae-dependent mechanism, while the presence of $\mathrm{M} \beta \mathrm{CD}$ appeared to enhance the cell uptake of NIM nanomicelles in the endocytosis study. Considering the similar effect of lipid raft on viruses,${ }^{35}$ we hypothesize that the disruption of the lipid raft/caveolae structure might facilitate the fusion of the recycling endosome compartment with the plasma membrane and trigger the increase of exocytosis. To explore the impact of COX inhibitor on the exocytosis of NIM nanomicelles, indomethacin was selected as the inhibitor of COX, which did not show a significantly different level of NIM exocytosis compared to the control $(P>0.05)$.

Furthermore, we looked at the effect of temperature on the exocytosis of NIM nanomicelles by measuring the intracellular NIM nanomicelles when reincubated with HBSS-only medium. The amount of NIM nanomicelles exocytosed at $4^{\circ} \mathrm{C}$ was significantly lower than that at $37^{\circ} \mathrm{C}(P<0.05$; Figure 5). This indicates that the exocytosis process of NIM nanomicelles was largely energy independent.

\section{Transport of NIM nanomicelles across Caco- 2 cell monolayer}

Figure 6A shows the Transwell ${ }^{\circledR}$ cell culture chamber. Figure $6 \mathrm{~B}$ shows the effects of NIM formulations on the transport of NIM across the Caco- 2 cell monolayer. Because of transport across the Caco- 2 cell monolayer, the cumulative transport of NIM nanomicelles from the apical to the basal side was significantly enhanced as compared to transport of Nimotop ${ }^{\circledR}(P<0.05)$. The nanomicelle formulation significantly increased the transport of NIM across the Caco-2 cell monolayer, which could be attributed to the increased accessibility of NIM nanomicelles to the cell surface and the enhanced cell uptake.

Figure $6 \mathrm{C}$ shows the TEM images of micelle-containing media collected from the apical and basolateral sides of the Caco-2 cell monolayer 1 hour after applying NIM nanomicelles 


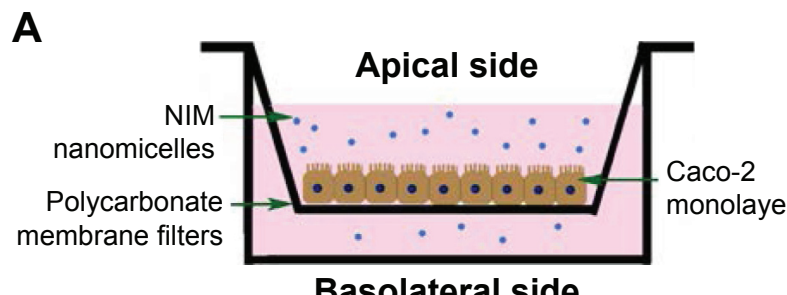

B

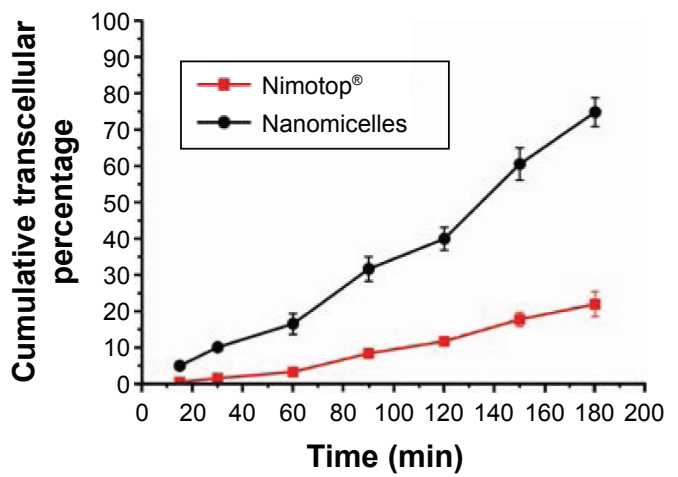

C

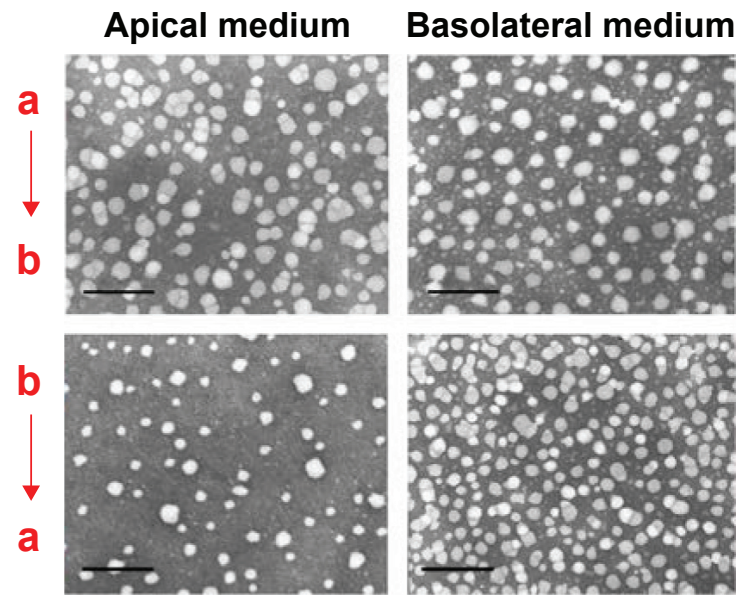

Figure 6 Transport of NIM nanomicelles across Caco-2 cell monolayer.

Notes: (A) Caco-2 cell monolayer model for human intestinal drug absorption study; (B) cumulative transport of NIM across the Caco-2 cell monolayers after applying NIM nanomicelles and Nimotop ${ }^{\circledR}$. (C) TEM images of media collected from the apical (a) and basolateral (b) sides of Caco-2 cell monolayers after incubation with NIM nanomicelles. Scale bar $=100 \mathrm{~nm}$; magnification was 100,000x. Data represent mean \pm SD $(n=3)$.

Abbreviations: NIM, nimodipine; TEM, transmission electron microscopy; SD, standard deviation; min, minutes.

in the apical or basolateral chamber. Near-spherical particles of $\sim 13 \mathrm{~nm}$ were observed on the basolateral side. The particle size of micelles at the basolateral side remained similar compared with those observed at the apical side. These results demonstrated that NIM nanomicelles either were intactly exocytosed across the Caco-2 cell monolayer or underwent rapid reassembly after exocytosis. The number of particles transported from apical to basolateral side $(\mathrm{A} \rightarrow \mathrm{B})$ was more than those transported from basolateral to apical side $(B \rightarrow A)$, which can be explained by the different proteins in the apical and basolateral membranes of polar epithelial cells. ${ }^{36}$

The TEER values of the monolayers were routinely monitored before and during the experiment. TEER value $>700 \Omega \cdot \mathrm{cm}^{2}$ was included as an indicator for the integrity and fluidity of Caco- 2 cell monolayers. After exposure to NIM nanomicelles for 3 hours, the TEER value decreased to $565 \pm 31 \Omega \cdot \mathrm{cm}^{2}$. This implied that the tight junctions of cell manolayers were not interrupted and the fluidity of the cell membrane was increased by the NIM nanomicelles, which could be advantageous in promoting intestinal permeability and cellular uptake in enterocytes.

\section{Absorption of NIM nanomicelles in the Gl tract}

The intestinal absorption of NIM nanomicelles was significantly changed with the site of the intestinal segments and the time points after oral administration. At 0.5 hour after oral administration, the tissue concentration of NIM nanomicelles was the highest in the duodenum, successively decreasing from the duodenum to the ileum (Figure 7A). Then, the NIM concentration from NIM nanomicelles in duodenum and jejunum was continuously reduced with the prolongation of the time intervals, while that in the ileum first reached the highest level at 1.0 hour (Figure 7B) and then was significantly reduced $(P<0.05)$. The distribution of NIM nanomicelles in the intestine could be dependent on the GI hydrodynamics, gastric emptying rate and forces, intestinal transit time and flow rate, etc. Compared with tablets, the NIM concentration from NIM nanomicelles was obviously improved 3.13- and 2.25-fold in duodenum and jejunum at 1 hour, but not statistically promoted in these intestinal segments at 4.0 hours $(P>0.05)$, as shown in Figure 7C. These results implied that the absorption of NIM nanomicelles in the intestine membrane was significantly improved by the self-assembled nanocarrier.

Moreover, the content of NIM nanomicelles in the intestine was visualized under a multimodal imaging system. The hydrophobic fluorescent dye DiR was almost entirely encapsulated into the nanocarrier, and the particle size of the fluorescent nanomicelles was $13.02 \pm 1.24 \mathrm{~nm}$ (PDI $=0.121$ ), which was comparable with the size of NIM nanomicelles. The ex vivo images showed that a strong DiR fluorescence 
A

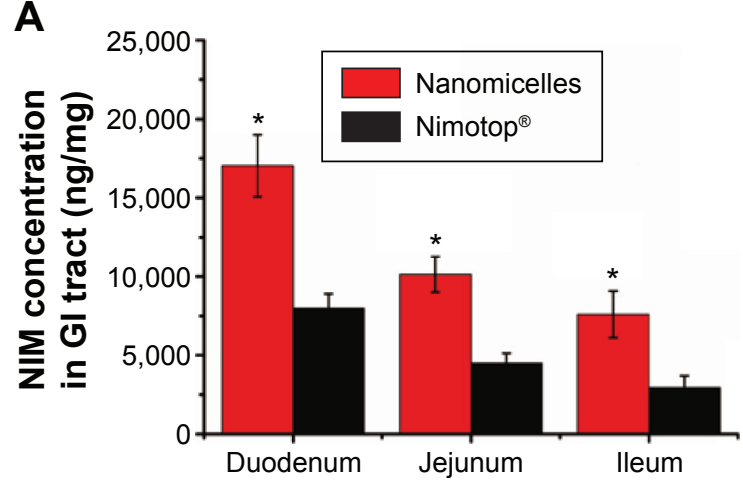

C

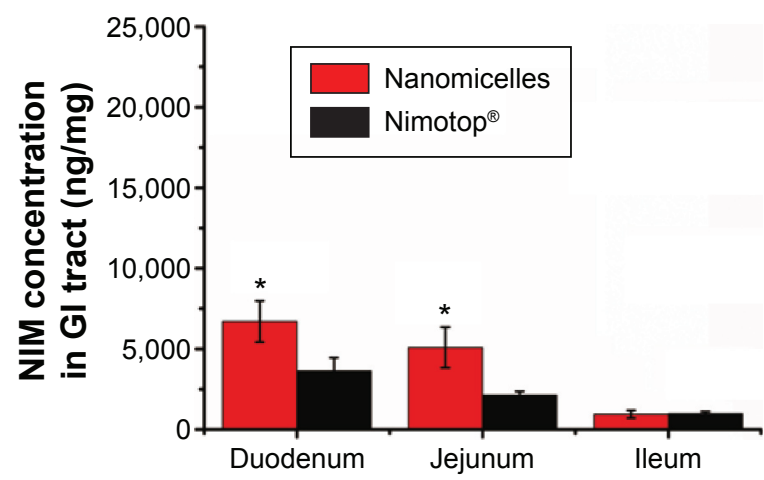

B

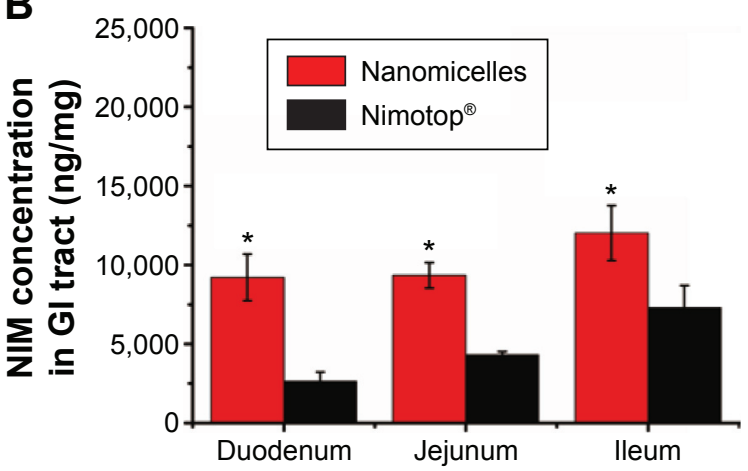

D

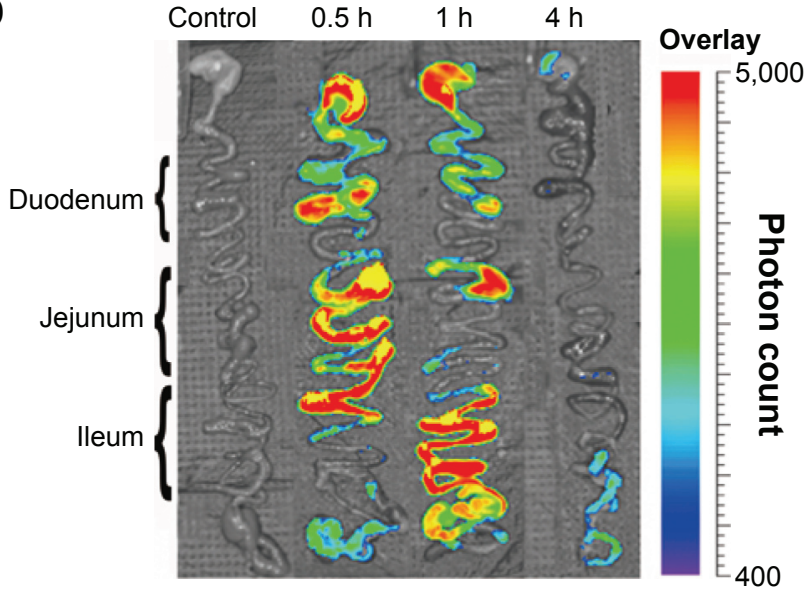

Figure 7 Absorption of NIM nanomicelles in the intestine at different time points after oral administration in rats.

Notes: (A) 0.5 hour; (B) I hour; and (C) 4.0 hours. (D) The ex vivo optical images of DiR-loaded nanomicelles in duodenum, jejunum, and ileum at 0.5 hour, I hour, and 4.0 hours after oral administration. Data represent mean \pm SD $(n=3) . * P<0.05$, versus Nimotop ${ }^{\circledR}$.

Abbreviations: DiR, I,I'-dioctadecyl-3,3,3',3'-tetramethylindotricarbocyanine iodide; GI, gastrointestinal; NIM, nimodipine; SD, standard deviation; min, minutes.

was observed in duodenum and jejunum, but this hardly appeared in ileum, which confirmed the uptake of nanomicelles in the enterocytes lining the intestine. These images further indicated that nanomicelles were mainly absorbed in the proximal intestine at 0.5 hour after oral administration, which was in accordance with the quantitative measurement results (Figure 7D).

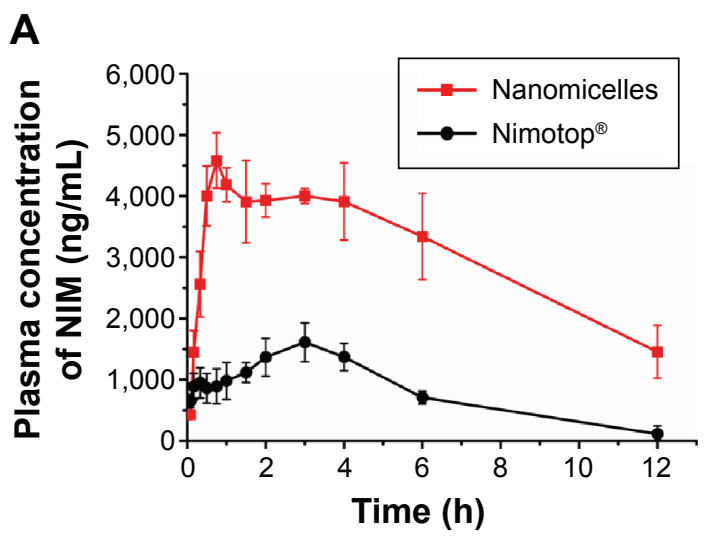

\section{Pharmacokinetics and biodistribution of NIM nanomicelles}

Following oral administration, micelles and Nimotop ${ }^{\circledR}$ showed very different pharmacokinetic profiles of NIM in rats. At each given time point, the NIM concentration in rats treated with NIM nanomicelles was significantly higher than that of $\operatorname{Nimotop}^{\circledR}(P<0.05)$ (Figure 8A).

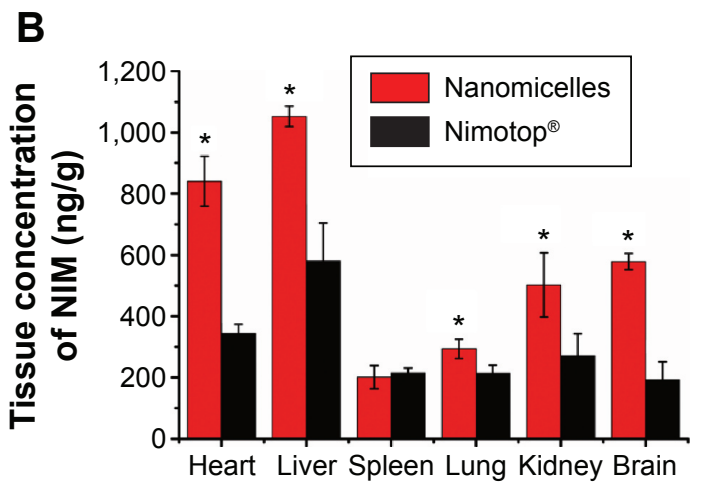

Figure 8 Pharmacokinetics and biodistribution of NIM nanomicelles.

Notes: (A) The plasma concentration-time curve of NIM in rats treated with NIM nanomicelles or Nimotop ${ }^{\circledR}$. (B) NIM concentration in various tissues in mice after oral administration of NIM nanomicelles and Nimotop ${ }^{\circledR}$. Data represent mean \pm SD $(n=5)$. ${ }^{*} P<0.05$, versus Nimotop ${ }^{\circledR}$.

Abbreviations: NIM, nimodipine; SD, standard deviation; h, hours. 
Table 4 Pharmacokinetic parameters of NIM nanomicelles and Nimotop ${ }^{\circledR}$ in rats

\begin{tabular}{llllll}
\hline Formulation & $\boldsymbol{C}_{\max }(\mathrm{ng} / \mathrm{mL})$ & $\boldsymbol{T}_{\max }$ (hours) & $\boldsymbol{t}_{1 / 2}$ (hours) & AUC $_{0-\infty}$ (ng/mL-h) & Relative bioavailability \\
\hline Nimotop ${ }^{\circledR}$ & $2,564.2 \pm 373.1$ & $2.4 \pm 0.1$ & $3.0 \pm 0.2$ & $13,996.5 \pm 1,827.2$ & - \\
NIM nanomicelles & $5,201.7 \pm 467.5^{*}$ & $2.4 \pm 0.1$ & $6.5 \pm 0.4^{*}$ & $52,126.6 \pm 4,710.3^{*}$ & 3.72 \\
\hline
\end{tabular}

Notes: Each value represents mean $\pm S D(n=5)$. $* P<0.05$ versus Nimotop ${ }^{\circledR}$.

Abbreviations: AUC, area under the plasma concentration-time curve; $C L$, clearance; $C_{\max }$, the maximum concentration in plasma; NIM, nimodipine; SD, standard deviation; $t_{1 / 2}$, elimination half-life; $T_{\max }$, peak time.

The pharmacokinetic parameters of NIM were summarized in Table 4. Compared with Nimotop ${ }^{\circledR}$, NIM nanomicelles showed significantly higher $C_{\max }(5,201.7 \pm 67.5 \mathrm{ng} / \mathrm{mL})$, $\mathrm{AUC}_{0-\infty}$ of $52,126.6 \pm 4,710.3 \mathrm{ng} \cdot \mathrm{h} / \mathrm{mL}(P<0.05)$. This indicates that oral absorption of lipophilic NIM was greatly enhanced by the self-assembled nanocarrier, which could be attributed to increased aqueous solubility of NIM, improved intestinal absorption, and subsequent intestinal lymphatic transport. HS15 was proven effective in enhancing the intestinal permeability and oral absorption of lipophilic drugs. ${ }^{13}$ Meanwhile, the nanometer-sized particles could improve the affinity and accessibility of nanomicelles to the intestinal membrane, thereby increasing its absorption in the intestine.

Subsequently, the distribution of NIM nanomicelles in mice was measured at 1 hour following oral administration (Figure 8B). The quantified measurements indicated that the orally administered NIM nanomicelles were mainly distributed in heart, lung, kidney, and brain in mice. In heart, the NIM nanomicelle group showed that the NIM concentration was significantly increased by 3.17 -fold. Interestingly, the NIM nanomicelle group showed a significantly higher level of NIM distribution in brain than for Nimotop ${ }^{\circledR}$ after oral administration $(P<0.05)$, which would be greatly beneficial for the treatment of neurovascular diseases.

\section{Conclusion}

In this study, Solutol ${ }^{\circledR}$ HS15-based NIM nanomicelles were fabricated using a one-step self-assembly method. NIM nanomicelles are proven to increase the solubility of NIM and facilitate the transport of NIM across the Caco-2 cell monolayer, with significantly improved bioavailability in mice after oral administration. The cell surface binding and uptake of NIM nanomicelles in Caco-2 cells were primarily energy dependent. In Caco-2 cells, the uptake of NIM nanomicelles was mediated by multiple pathways, including clathrin-dependent uptake, lipid raft/ caveolae-mediated endocytosis, and macropinocytosis. The exocytosis of NIM nanomicelles was probably regulated by the Golgi complex and ER. In summary, Solutol ${ }^{\circledR}$
HS15-based nanomicelles represent a promising delivery system for improving the oral bioavailability of poorly soluble drugs.

\section{Acknowledgments}

This work was supported by the National Science and Technology Major Project of China (2014ZX09507001002), the National Basic Research Program of China (973 program, 2013CB932504), and the National Natural Science Foundation of China (81273443).

\section{Disclosure}

The authors report no conflicts of interest in this work.

\section{References}

1. Basalious EB, Shamma RN. Novel self-assembled nano-tubular mixed micelles of Pluronics P123, Pluronic F127 and phosphatidylcholine for oral delivery of nimodipine: in vitro characterization, ex vivo transport and in vivo pharmacokinetic studies. Int J Pharm. 2015;493(1-2): 347-356.

2. Huang S, Yu X, Yang L, et al. The efficacy of nimodipine drug delivery using mPEG-PLA micelles and mPEG-PLA/TPGS mixed micelles. Eur J Pharm Sci. 2014;63:187-198.

3. Fu Q, Sun J, Ai X, et al. Nimodipine nanocrystals for oral bioavailability improvement: role of mesenteric lymph transport in the oral absorption. Int J Pharm. 2013;448(1):290-297.

4. Xiong R, Lu W, Li J, Wang P, Xu R, Chen T. Preparation and characterization of intravenously injectable nimodipine nanosuspension. Int J Pharm. 2008;350(1-2):338-343.

5. Soliman GM, Sharma R, Choi AO, et al. Tailoring the efficacy of nimodipine drug delivery using nanocarriers based on A2B miktoarm star polymers. Biomaterials. 2010;31(32):8382-8392.

6. Jijun F, Lili Z, Tingting G, Xing T, Haibing H. Stable nimodipine tablets with high bioavailability containing NM-SD prepared by hot-melt extrusion. Powder Technol. 2010;204(2-3):214-221.

7. Riekes MK, Caon T, da Silva J, et al. Enhanced hypotensive effect of nimodipine solid dispersions produced by supercritical $\mathrm{CO} 2$ drying. Powder Technol. 2015;278:204-210.

8. Fu Q, Kou L, Gong C, et al. Relationship between dissolution and bioavailability for nimodipine colloidal dispersions: the critical size in improving bioavailability. Int J Pharm. 2012;427(2):358-364.

9. Fu Q, Sun J, Zhang D, et al. Nimodipine nanocrystals for oral bioavailability improvement: preparation, characterization and pharmacokinetic studies. Colloids Surf B Biointerfaces. 2013;109:161-166.

10. Subedi RK, Kang KW, Choi H-K. Preparation and characterization of solid lipid nanoparticles loaded with doxorubicin. Eur J Pharm Sci. 2009; 37(3-4):508-513.

11. Seo SW, Han HK, Chun MK, Choi HK. Preparation and pharmacokinetic evaluation of curcumin solid dispersion using Solutol(R) HS15 as a carrier. Int J Pharm. 2012;424(1-2):18-25. 
12. Lian XH, Dong JX, Zhang JJ, et al. Soluplus ${ }^{\circledR}$ based 9-nitrocamptothecin solid dispersion for peroral administration: preparation, characterization, in vitro and in vivo evaluation. Int J Pharm. 2014;477:399-407.

13. Bravo González RC, Huwyler J, Walter I, Mountfield R, Bittner B. Improved oral bioavailability of cyclosporin A in male Wistar rats. Int J Pharm. 2002;245(1-2):143-151.

14. Murgia S, Fadda P, Colafemmina G, et al. Characterization of the Solutol ${ }^{\mathbb{R}}$ HS15/water phase diagram and the impact of the $\Delta^{9}$-tetrahydrocannabinol solubilization. J Colloid Interface Sci. 2013;390:129-136.

15. Duhem N, Rolland J, Riva R, et al. Tocol modified glycol chitosan for the oral delivery of poorly soluble drugs. Int J Pharm. 2012;423(2): 452-460.

16. Dou J, Zhang H, Liu X, Zhang M, Zhai G. Preparation and evaluation in vitro and in vivo of docetaxel loaded mixed micelles for oral administration. Colloids Surf B Biointerfaces. 2014;114:20-27.

17. Zhao S, Dai W, He B, et al. Monitoring the transport of polymeric micelles across MDCK cell monolayer and exploring related mechanisms. J Control Release. 2012;158(3):413-423.

18. Ivanov AI, Nusrat A, Parkos CA. Endocytosis of epithelial apical junctional proteins by a clathrin-mediated pathway into a unique storage compartment. Mol Biol Cell. 2004;15(1):176-188.

19. Huang J, Li F, Wu J, Yang F. White spot syndrome virus enters crayfish hematopoietic tissue cells via clathrin-mediated endocytosis. Virology. 2015;486:35-43.

20. Yao HJ, Ju RJ, Wang XX, et al. The antitumor efficacy of functional paclitaxel nanomicelles in treating resistant breast cancers by oral delivery. Biomaterials. 2011;32(12):3285-3302.

21. Rodriguez DA, Tapia JC, Fernandez JG, et al. Caveolin-1-mediated suppression of cyclooxygenase-2 via a beta-catenin-Tcf/Lef-dependent transcriptional mechanism reduced prostaglandin E2 production and survivin expression. Mol Biol Cell. 2009;20(8):2297-2310.

22. Simonsen A, Wurmser AE, Emr SD, Stenmark H. The role of phosphoinositides in membrane transport. Curr Opin Cell Biol. 2001;13: 485-492.

23. Duncan R, Richardson SC. Endocytosis and intracellular trafficking as gateways for nanomedicine delivery: opportunities and challenges. Mol Pharm. 2012;9(9):2380-2402.
24. Decuzzi P, Ferrari M. The role of specific and non-specific interactions in receptor-mediated endocytosis of nanoparticles. Biomaterials. 2007; 28(18):2915-2922.

25. Head BP, Patel HH, Insel PA. Interaction of membrane/lipid rafts with the cytoskeleton: impact on signaling and function: membrane/ lipid rafts, mediators of cytoskeletal arrangement and cell signaling. Biochim Biophys Acta. 2014;1838(2):532-545.

26. Kerr MC, Teasdale RD. Defining macropinocytosis. Traffic. 2009; 10(4):364-371.

27. Liou JY, Deng WG, Gilroy DW, Shyue SK, Wu KK. Colocalization and interaction of cyclooxygenase-2 with caveolin-1 in human fibroblasts. J Biol Chem. 2001;276(37):34975-34982.

28. Tsujita K, Itoh T. Phosphoinositides in the regulation of actin cortex and cell migration. Biochim Biophys Acta. 2015;1851(6):824-831.

29. Tzafriri AR, Wu D, Edelman ER. Analysis of compartmental models of ligand-induced endocytosis. J Theor Biol. 2004;229(1):127-138.

30. Apodaca G. Endocytic traffic in polarized epithelial cells: role of the actin and microtubule cytoskeleton. Traffic. 2001;2:149-159.

31. Kis O, Zastre JA, Hoque MT, Walmsley SL, Bendayan R. Role of drug efflux and uptake transporters in atazanavir intestinal permeability and drug-drug interactions. Pharm Res. 2012;30(4):1050-1064.

32. Burd CG. Physiology and pathology of endosome-to-Golgi retrograde sorting. Traffic. 2011;12(8):948-955.

33. Feng Y, Yu S, Lasell TKR, et al. Exo1: a new chemical inhibitor of the exocytic pathway. Proc Natl Acad Sci U S A. 2003;100(11):6469-6474.

34. Duan J, Xie Y, Luo H, Li G, Wu T, Zhang T. Transport characteristics of isorhamnetin across intestinal Caco-2 cell monolayers and the effects of transporters on it. Food Chem Toxicol. 2014;66:313-320.

35. Barman S, Nayak DP. Lipid raft disruption by cholesterol depletion enhances influenza A virus budding from MDCK cells. J Virol. 2007; 81(22):12169-12178.

36. Browna PS, Wanga E, Aroeti B, Chapin SJ, Mostov KE, Dunn KW. Definition of distinct compartments in polarized Madin-Darby canine kidney (MDCK) cells for membrane-volume sorting, polarized sorting and apical recycling. Traffic. 2000;1:124-140.
International Journal of Nanomedicine

\section{Publish your work in this journal}

The International Journal of Nanomedicine is an international, peerreviewed journal focusing on the application of nanotechnology in diagnostics, therapeutics, and drug delivery systems throughout the biomedical field. This journal is indexed on PubMed Central,

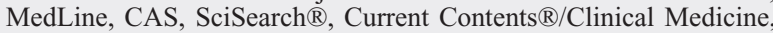

\section{Dovepress}

Journal Citation Reports/Science Edition, EMBase, Scopus and the Elsevier Bibliographic databases. The manuscript management system is completely online and includes a very quick and fair peer-review system, which is all easy to use. Visit http://www.dovepress.com/ testimonials.php to read real quotes from published authors. 